\title{
MAGNETOHYDRODYNAMIC FLOW OF A MICROPOLAR FLUID IN A CIRCULAR PIPE WITH HALL EFFECTS
}

\author{
D. SRINIVASACHARYA ${ }^{\otimes 1}$ and MEKONNEN SHIFERAW ${ }^{2}$
}

(Received 5 March, 2009; revised 13 November, 2009)

\begin{abstract}
Steady magnetohydrodynamic flow of an incompressible micropolar fluid through a pipe of circular cross-section is studied by considering Hall and ionic effects. The fluid motion is due to a constant pressure gradient, and an external uniform magnetic field directed perpendicular to the flow direction is applied. Expressions for the velocity, microrotation, skin friction and flow rate are obtained. The effects of the micropolar parameter, magnetic parameter, Hall parameter and ion-slip parameter on the velocity, microrotation, skin friction and flow rate are discussed.
\end{abstract}

2000 Mathematics subject classification: primary 76A05; secondary 76W05.

Keywords and phrases: magnetohydrodynamics, micropolar fluid, circular pipe, Hall effects.

\section{Introduction}

In recent years, several simple flow problems associated with classical hydrodynamics have received attention within the more general context of magnetohydrodynamics (MHD). Several investigators have extended many of the available hydrodynamic solutions to include the effects of magnetic fields for those cases where the fluid is electrically conducting. Also, the study of non-Newtonian fluid flows has received much attention from researchers because of the variety of applications in biology, physiology, technology and industry, such as blood flow through arteries, vasomotion of small blood vessels, drilling of oil and gas wells, production of synthetic fibers, foodstuffs, the extrusion of molten plastics, liquid metals and alloys, plasma, clay coating and emulsions and elastomers. In addition, the effects of a magnetic field on a non-Newtonian fluid have great importance in engineering applications, such as MHD generators, accelerators, aerodynamic heating, electrostatic precipitation,

\footnotetext{
${ }^{1}$ Department of Mathematics, National Institute of Technology, Warangal 506 004, India; e-mail: dsc@nitw.ac.in, dsrinivasacharya@yahoo.com.

${ }^{2}$ Department of Mathematics, Arba Minch University, PO Box 72, Arba Minch, Ethiopia; e-mail: mekk_aya@yahoo.com.

(C) Australian Mathematical Society 2010, Serial-fee code 1446-1811/2010 \$16.00
} 
polymer technology, the petroleum industry, purification of crude oil and fluid droplet sprays, plasma studies and geothermal energy excitations.

Unlike many non-Newtonian fluid models which describe the nonlinear relationship between stress and the rate of strain, the fluid model introduced by Eringen [7] allows for some microscopic effects arising from the local structure and micromotion of the fluid elements. The micropolar fluids sustain couple stresses and include the classical Newtonian fluid as a special case. The model of a micropolar fluid represents fluids consisting of rigid, randomly oriented (or spherical) particles suspended in a viscous medium where the deformation of the particles is ignored. Fluids containing certain additives, polymeric fluids and animal blood are examples of micropolar fluids. A detailed survey of microcontinuum fluid mechanics with several applications has been presented by Ariman et al. [2]. The mathematical theory of micropolar fluids and applications to the theory of lubrication and the theory of porous media are presented by Lukaszewicz [9].

Several investigators have made theoretical studies of micropolar fluid flow in the presence of a transverse magnetic field. Kasiviswanathan and Gandhi [8] studied a class of exact solutions for the MHD flow of a micropolar fluid confined between two infinite, insulated, parallel, noncoaxially rotating disks. Bhargava et al. [5] analysed the effect of temperature-dependent heat sources on the fully developed free convection of an electrically conducting micropolar fluid between two parallel porous vertical plates in a transverse magnetic field. Ahmadi and Shahinpoor [1] studied the criteria for universal stability of the unsteady motion of an incompressible, electrically conducting linear micropolar fluid.

The Hall and ion-slip terms in Ohm's law have been ignored in most of the MHD flow problems considered thus far. However, in the presence of a strong magnetic field, the influence of Hall current and ion slip is important. Tani [12] studied the Hall effect on the steady motion of electrically conducting viscous fluid in channels. Hall and ion-slip effects in MHD Couette flow with heat transfer have been considered by Soundelgekar et al. [11]. Attia [3, 4] analysed the Hall effects on velocity and temperature fields of unsteady Hartmann flows and on the transient flow of dusty viscous incompressible electrically conducting non-Newtonian Bingham fluid through a circular pipe. Seddeek [10] studied the effects of Hall and ion-slip currents on a magneto-micropolar fluid and heat transfer over a nonisothermal stretching sheet with suction and blowing.

In this paper, the steady incompressible electrically conducting micropolar fluid flow in a circular pipe is studied by considering Hall and ion-slip current effects. The expressions for velocity, microrotation component, flow rate and skin friction (shear stress at the wall) are obtained and their variation with respect to various parameters is studied.

\section{Formulation of the problem}

Consider the steady axisymmetric flow of an incompressible electrically conducting micropolar fluid through an infinitely long circular pipe of radius $a$ driven by a constant pressure gradient. Choose the cylindrical polar coordinate system $(r, \theta, z)$ with $z$-axis 
in the direction of motion. A uniform magnetic field is applied perpendicular to the flow direction. The Hall and ion-slip current is taken into consideration. A very small magnetic Reynolds number is assumed and therefore the induced magnetic field is neglected. Within the framework of these assumptions, the equations that govern the MHD flow in the absence of both body force (force per unit volume) and body couple (moment per unit volume) are

$$
\begin{gathered}
\frac{d p}{d z}+\frac{\kappa}{r} \frac{d}{d r}(r \omega)+(\mu+\kappa) \frac{1}{r} \frac{d}{d r}\left(r \frac{d u}{d r}\right)-\frac{\sigma B_{0}^{2}\left(1+B_{i} B_{h}\right)}{\left(1+B_{i} B_{h}\right)^{2}+B_{h}^{2}} u=0, \\
-2 \kappa \omega-\kappa \frac{d u}{d r}+\gamma \frac{d}{d r}\left\{\frac{1}{r} \frac{d}{d r}(r \omega)\right\}=0
\end{gathered}
$$

where $u(r)$ is the velocity component, $\omega(r)$ the component of microrotation, $\rho$ the density, $p$ the fluid pressure, $\mu$ the viscosity coefficient, $\kappa$ the vortex viscosity, $\gamma$ the spin-gradient viscosity, $\sigma$ the electrical conductivity of the fluid, $B_{i}$ the ion-slip parameter, $B_{0}$ the magnetic induction, and for Hall factor $\eta$, the Hall parameter is $B_{h}=\sigma \eta B_{0}$.

In the limit as $\kappa \rightarrow 0$, Equation (2.1) is uncoupled from (2.2) and reduces to the case of a Newtonian fluid.

Defining $p_{0}=d p / d z$, introducing the nondimensional variables

$$
r=a \tilde{r}, \quad u=\frac{p_{0} a^{2}}{\mu} \tilde{u}, \quad \omega=\frac{p_{0} a}{\mu} \tilde{\omega},
$$

into Equations (2.1) and (2.2) and dropping tildes, we obtain

$$
\begin{aligned}
-1+\frac{N}{1-N} \frac{1}{r} & \frac{d}{d r}(r \omega)+\frac{1}{1-N} \frac{1}{r} \frac{d}{d r}\left(r \frac{d u}{d r}\right)-\frac{H_{a}^{2}\left(1+B_{i} B_{h}\right)}{\left(1+B_{i} B_{h}\right)^{2}+B_{h}^{2}} u=0, \\
& -2 \omega-\frac{d u}{d r}+\frac{2-N}{m^{2}}\left[\frac{d}{d r}\left\{\frac{1}{r} \frac{d}{d r}(r \omega)\right\}\right]=0,
\end{aligned}
$$

where $N=\kappa /(\mu+\kappa)$ is the coupling number $(0 \leq N<1)[6]$, which defines the coupling of the linear (2.3) and angular momentum equations (2.4). When $N$ is identically zero, the equations of linear and angular momentum are decoupled and the equation of linear momentum reduces to the classical Navier-Stokes equation. The Hartmann number, $H_{a}=B_{0} a \sqrt{\sigma / \mu}$, describes the effective strength of the magnetic induction and, following Eringen [7], $m^{2}=a^{2} \kappa(2 \mu+\kappa) /(\gamma(\mu+\kappa))$ is the micropolar parameter.

The boundary conditions are the usual no-slip and hyper-stick conditions

$$
\begin{aligned}
u=0 \quad \text { at } r=a, \\
\omega=0 \quad \text { at } r=a, \\
\frac{d u}{d r}=0 \quad \text { at } r=0,
\end{aligned}
$$

and $u$ and $\omega$ are finite at $r=0$. 


\section{Solution of the problem}

Differentiating Equation (2.3) with respect to $r$ and substituting in Equation (2.4), we get

$$
\omega=\frac{2-N}{2 N m^{2}} \frac{d}{d r}\left(D^{2} u\right)+\left[-\frac{1}{2}+\frac{(2-N)(1-N)}{2 m^{2} N} \frac{H_{a}^{2}\left(1+B_{i} B_{h}\right)}{\left(1+B_{i} B_{h}\right)^{2}+B_{h}^{2}}\right] \frac{d u}{d r},
$$

where

$$
D^{2}=\frac{d}{d r}\left\{\frac{1}{r} \frac{d}{d r}(r \omega)\right\} .
$$

Substituting Equation (3.1) in Equation (2.3), we obtain

$$
\left[D^{4}-\left(\alpha_{1}^{2}+\alpha_{2}^{2}\right) D^{2}+\alpha_{1}^{2} \alpha_{2}^{2}\right] u=\frac{-2(1-N) m^{2}}{2-N},
$$

where

$$
\begin{aligned}
\alpha_{1}^{2}+\alpha_{2}^{2} & =m^{2}+(1-N) \frac{H_{a}^{2}\left(1+B_{i} B_{h}\right)}{\left(1+B_{i} B_{h}\right)^{2}+B_{h}^{2}}, \\
\alpha_{1}^{2} \alpha_{2}^{2} & =\frac{2(1-N) m^{2}}{2-N} \frac{H_{a}^{2}\left(1+B_{i} B_{h}\right)}{\left(1+B_{i} B_{h}\right)^{2}+B_{h}^{2}} .
\end{aligned}
$$

The solution of Equation (3.2) is

$$
u(r)=C_{1} I_{0}\left(\alpha_{1} r\right)+C_{2} K_{0}\left(\alpha_{1} r\right)+C_{3} I_{0}\left(\alpha_{2} r\right)+C_{4} K_{0}\left(\alpha_{2} r\right)-\frac{2(1-N) m^{2}}{\alpha_{1}^{2} \alpha_{2}^{2}(2-N)},
$$

where $I_{0}$ and $K_{0}$ are modified zeroth-order Bessel functions of the first and second kind respectively and $C_{1}, C_{2}, C_{3}$ and $C_{4}$ are arbitrary constants. Substituting Equation (3.4) into Equation (3.1) gives

$$
\omega(r)=C_{\alpha}\left[C_{1} I_{1}\left(\alpha_{1} r\right)+C_{2} K_{1}\left(\alpha_{1} r\right)\right]+C_{\beta}\left[C_{3} I_{1}\left(\alpha_{2} r\right)+C_{4} K_{1}\left(\alpha_{2} r\right)\right],
$$

where

$$
C_{\alpha}=\frac{\alpha_{1}\left[\alpha_{2}^{2}(2-N) 2 m^{2}\right]}{2 N m^{2}}, \quad C_{\beta}=\frac{\alpha_{2}\left[\alpha_{1}^{2}(2-N) 2 m^{2}\right]}{2 N m^{2}}
$$

and $I_{1}$ and $K_{1}$ are modified first-order Bessel functions of the first and second kind, respectively. The arbitrary constants $C_{2}$ and $C_{4}$ are both zero as we require $\omega$ to be finite for $r=0$. Constants $C_{1}$ and $C_{3}$ are determined using Equations (2.5) and (2.6) and the solutions are

$$
\begin{aligned}
& u(r)=\frac{2(1-N) m^{2}}{\alpha_{1}^{2} \alpha_{2}^{2}(2-N)} \frac{C_{\alpha} I_{1}\left(\alpha_{1}\right) I_{0}\left(\alpha_{2} r\right) C_{\beta} I_{1}\left(\alpha_{2}\right) I_{0}\left(\alpha_{1} r\right)}{\left[C_{\alpha} I_{0}\left(\alpha_{2}\right) I_{1}\left(\alpha_{1}\right)-C_{\beta} I_{0}\left(\alpha_{1}\right) I_{1}\left(\alpha_{2}\right)\right]}, \\
& \omega(r)=\frac{2(1-N) m^{2}}{\alpha_{1}^{2} \alpha_{2}^{2}(2-N)} \frac{C_{\alpha} C_{\beta}\left[I_{1}\left(\alpha_{1}\right) I_{1}\left(\alpha_{2} r\right)-I_{1}\left(\alpha_{2}\right) I_{1}\left(\alpha_{1} r\right)\right]}{\left[C_{\alpha} I_{0}\left(\alpha_{2}\right) I_{1}\left(\alpha_{1}\right)-C_{\beta} I_{0}\left(\alpha_{1}\right) I_{1}\left(\alpha_{2}\right)\right]} .
\end{aligned}
$$


(a)

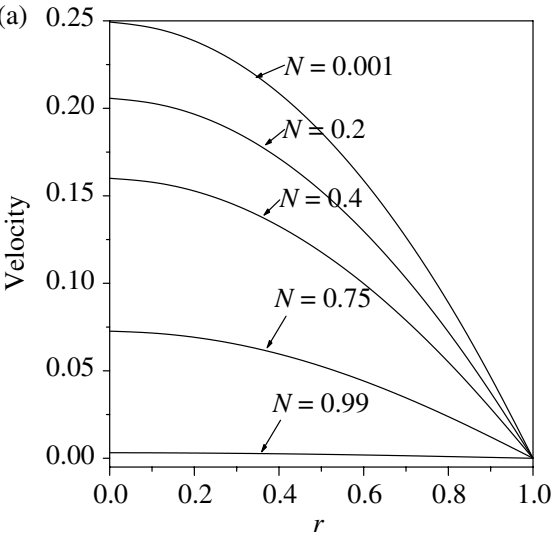

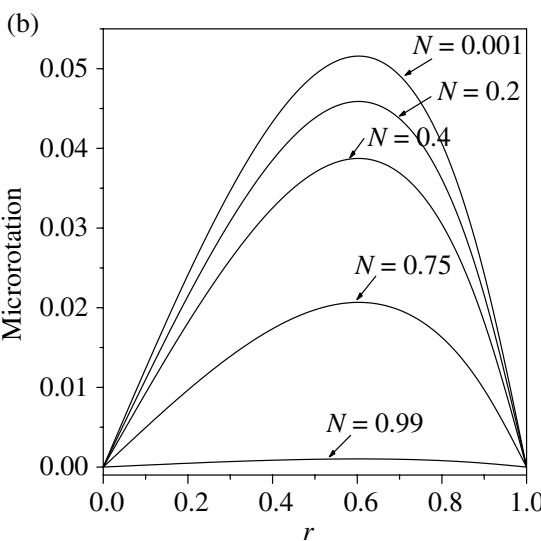

FIGURE 1. Effect of $N$ on (a) velocity given by (3.6) and (b) microrotation given by (3.7) of a fluid with $B_{i}=0.2, B_{h}=2, H_{a}=0.2$ and $m=2.5$.

From Equation (3.7) we observe that $\omega=0$ on $r=0$, which is consistent with the expectation that microrotation is zero on the $z$ axis [7].

The dimensionless flow rate defined as $Q=\int_{0}^{1} 2 r u(r) d r$ can be obtained from Equation (3.6) in the form

$$
Q=\frac{2(1-N) m^{2}}{\alpha_{1}^{2} \alpha_{2}^{2}(2-N)}\left(2 \frac{\alpha_{1} C_{\alpha} I_{1}\left(\alpha_{1}\right) I_{0}\left(\alpha_{2}\right)-\alpha_{2} C_{\beta} I_{1}\left(\alpha_{2}\right) I_{1}\left(\alpha_{1}\right)}{\alpha_{1} \alpha_{2}\left[C_{\alpha} I_{0}\left(\alpha_{2}\right) I_{1}\left(\alpha_{1}\right)-C_{\beta} I_{0}\left(\alpha_{1}\right) I_{1}\left(\alpha_{2}\right)\right]}-1\right) .
$$

The nonzero dimensionless shearing stresses are given by

$$
\tau_{r z}=\frac{1}{1-N} \frac{\partial u}{\partial r}+\frac{N}{1-N} \omega \quad \text { and } \quad \tau_{z r}=\frac{\partial u}{\partial r}-\frac{N}{1-N} \omega .
$$

\section{Results and discussion}

The effects of coupling number $(N)$, magnetic parameter $\left(H_{a}\right)$, Hall parameter $\left(B_{h}\right)$ and ion-slip parameter $\left(B_{i}\right)$ on velocity, microrotation, flow rate and skin friction of a fluid are evaluated numerically and the results are presented in Figures 1-4 and Tables 1-3. The coupling number $N$ characterizes the coupling of linear and rotational motion arising from the micromotion of the fluid molecules. Hence $N$ signifies the coupling between the Newtonian and rotational viscosities. With $N$ close to 1 the effect of microstructure becomes significant, whereas with $N$ close to 0 the individuality of the substructure is much less pronounced. As $\kappa$ tends to zero $N$ also tends to zero, micropolarity is lost and the fluid is expected to behave as a nonpolar fluid, and the expression for the velocity obtained in this paper reduces to its counterpart in classical Newtonian theory.

The predicted variation of velocity and microrotation with radius $(r)$ for different values of $N$ and for $B_{h}=2, B_{i}=0.2, H_{a}=0.2$ and $m=2.5$ is shown in Figure 1. 

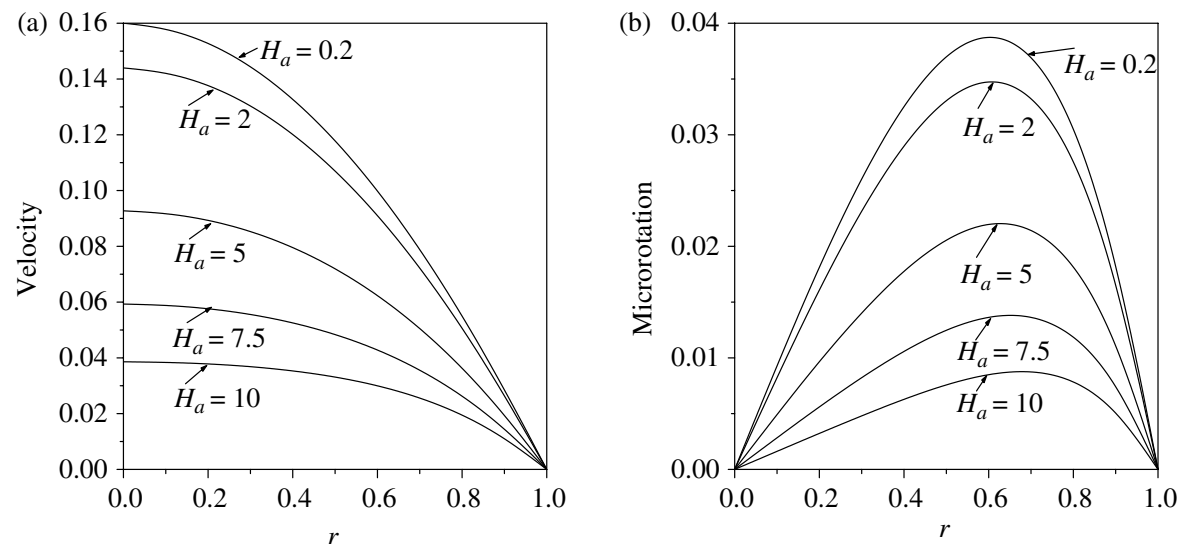

FIGURE 2. Effect of $H_{a}$ on (a) velocity given by (3.6) and (b) microrotation given by (3.7) of a fluid with $B_{i}=0.2, B_{h}=2, m=2.5$ and $N=0.4$.
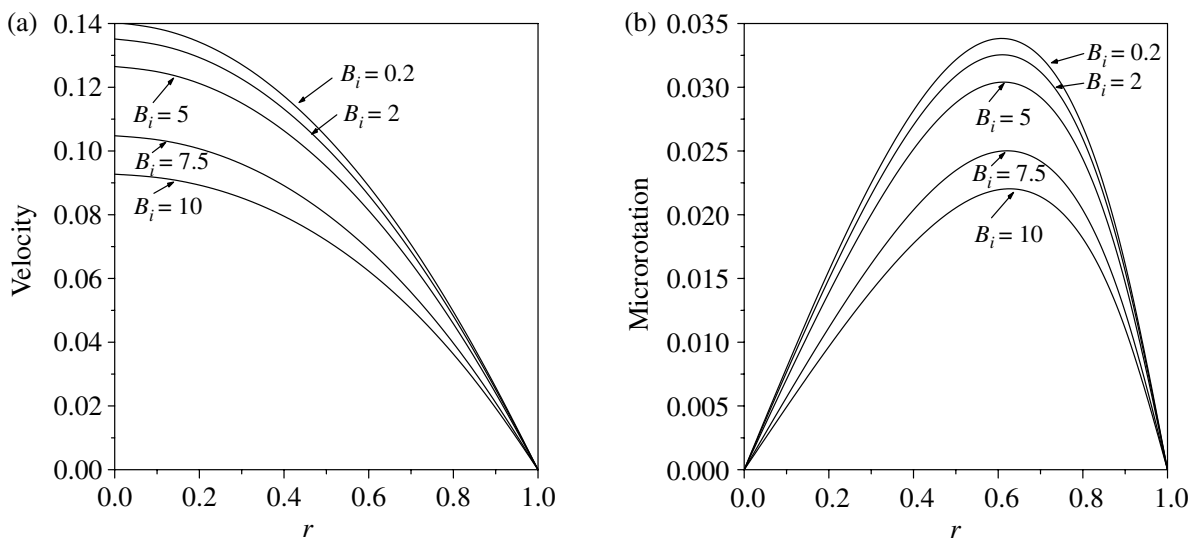

FIGURE 3. Effect of $B_{i}$ on (a) velocity given by (3.6) and (b) microrotation given by (3.7) of a fluid with $H_{a}=5, B_{h}=2, m=2.5$ and $N=0.4$.

Velocity and microrotation decrease as $N$ increases. In the limit as $N \rightarrow 0$, Equations (2.3) and (2.4) reduce to the corresponding equations for a viscous fluid. Hence, it can be observed that the velocity in the case of micropolar fluid is less than that of viscous fluid. Furthermore, for fixed $N$ the microrotation increases and then decreases as radial distance from the axis increases.

The effect of $H_{a}$ on the velocity and microrotation is shown in Figure 2. Figure 2(a) shows that the velocity, as expected, becomes small for large values of $H_{a}$. This happens because the applied magnetic field gives rise to a resistive force and slows down the movement of the fluid in the pipe. Figure 2(b) shows decreasing angular velocity with increasing $H_{a}$. 

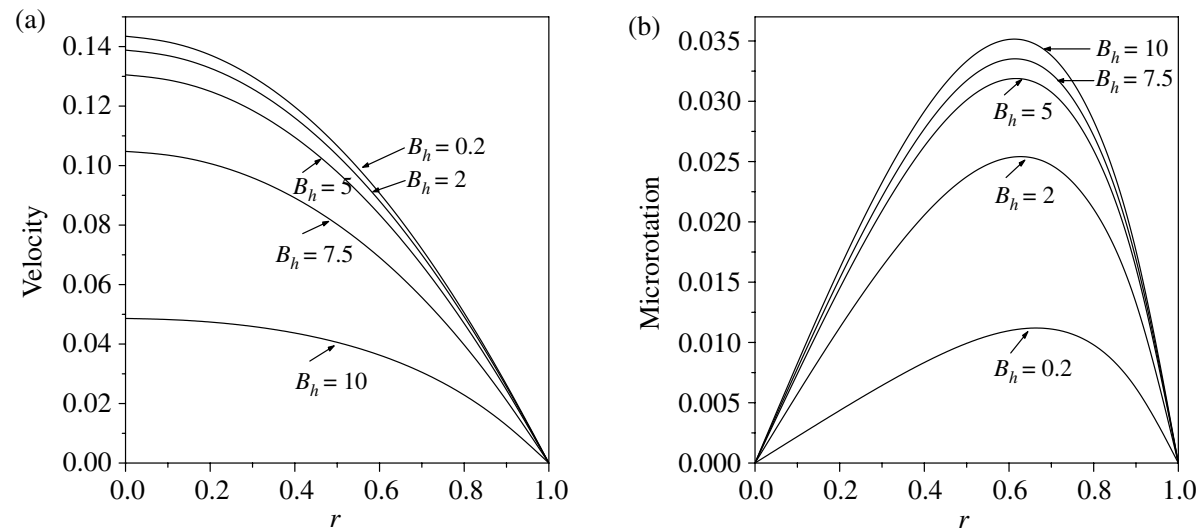

FIGURE 4. Effect of $B_{h}$ on (a) velocity given by (3.6) and (b) microrotation given by (3.7) of a fluid with $H_{a}=5, B_{i}=2, m=2.5$ and $N=0.4$.

TABLE 1 . The skin friction $(F)$ and flow rate $(Q)$ for $H_{a}=5, N=0.4, m=2.5$ and $B_{h}=2$.

\begin{tabular}{lcl}
\hline$B_{i}$ & skin friction & flow rate \\
\hline 0.2 & 0.21 & 0.049 \\
2.0 & 0.23 & 0.054 \\
5.0 & 0.26 & 0.064 \\
7.5 & 0.27 & 0.068 \\
10 & 0.28 & 0.070 \\
\hline
\end{tabular}

TABLE 2. The skin friction $(F)$ and flow rate $(Q)$ for $H_{a}=5, N=0.4, m=2.5$ and $B_{i}=2$.

\begin{tabular}{lcl}
\hline$B_{h}$ & skin friction & flow rate \\
\hline 0.2 & 0.15 & 0.028 \\
2.0 & 0.23 & 0.054 \\
5.0 & 0.26 & 0.066 \\
7.5 & 0.27 & 0.069 \\
10 & 0.28 & 0.071 \\
\hline
\end{tabular}

Figure 3 shows the velocity and microrotation profiles for different values of the ion-slip parameter $B_{i}$. Both velocity and microrotation profiles increase as $B_{i}$ increases. As $B_{i}$ increases the effective conductivity decreases, which in turn decreases the damping force on velocity, and hence the velocity increases.

Figure 4 shows the predicted effect of the Hall parameter $B_{h}$ on velocity and microrotation of a fluid. Velocity and microrotation both increase with Hall parameter. 
TABLE 3. The skin friction $(F)$ and flow rate $(Q)$ of a fluid with $H_{a}=5, B_{h}=2, m=2.5$ and $B_{i}=2$.

\begin{tabular}{lcl}
\hline$N$ & skin friction & flow rate \\
\hline 0.1 & 0.32 & 0.069 \\
0.3 & 0.26 & 0.060 \\
0.5 & 0.20 & 0.048 \\
0.7 & 0.13 & 0.033 \\
0.9 & 0.047 & 0.013 \\
\hline
\end{tabular}

Inclusion of $B_{h}$ in the model reduces the predicted effective conductivity of the fluid, resulting in a decrease in the resistive force caused by the magnetic field.

Table 1 presents the values of the flow rate and skin friction coefficient predicted for a fluid for a range of values of the ion-slip parameter. The flow rate and the skin friction increase as $B_{i}$ increases. These effects are consequences of the increase of the velocity of the fluid with increasing $B_{i}$.

Table 2 presents the values of the flow rate and skin friction coefficient predicted for a fluid for a range of values of $B_{h}$. The flow rate and the skin friction increase as $B_{h}$ increases. This is a consequence of the increase in the fluid velocity resulting from increasing $B_{h}$.

Table 3 anticipates that a micropolar fluid has reduced skin friction compared to that of a Newtonian fluid $(N \rightarrow 0)$. As $N$ increases, flow rate decreases.

\section{Conclusions}

The steady flow of conducting micropolar fluid through a pipe under the influence of an applied uniform magnetic field has been studied, considering Hall and ion-slip effects. Analytical solutions for the equation of motion have been obtained. The effects of magnetic field, the Hall parameter, the ion-slip parameter and Hartmann number on the velocity and microrotation have been investigated. Our results predict that increasing the magnetic field intensity decreases the velocity and the microrotation, while increasing the Hall and ion-slip parameter leads to an increase in both the velocity and the microrotation. It is predicted that as the ion-slip parameter and Hall parameter increase, both the flow rate and skin friction coefficient increase.

\section{References}

[1] G. Ahmadi and M. Shahinpoor, "Universal stability of magneto-micropolar fluid motions", Int. J. Eng. Sci. 12 (1974) 657-663.

[2] T. Ariman, M. A. Turk and N. D. Sylvester, "Micro continuum fluid mechanics-a review", Int. J. Eng. Sci. 11 (1973) 905-930.

[3] H. A. Attia, "Hall current effects on the velocity and temperature fields of unsteady Hartmann flow”, Canad. J. Phys. 76 (1998) 739-746. 
[4] H. A. Attia, "Hall effect on the flow of a dusty Bingham fluid in a circular pipe", Turkish J. Eng. Env. Sci. 30 (2006) 14-21.

[5] R. Bhargava, L. Kumar and H. S. Takhar, "Numerical solution of free convection MHD micropolar fluid flow between parallel porous vertical plates", Int. J. Eng. Sci. 41 (2003) 123-136.

[6] S. C. Cowin, "Polar fluids", Phys. Fluids 11 (1968) 1919-1927.

[7] A. C. Eringen, "The theory of micropolar fluids", J. Math. Mech. 16 (1966) 1-16.

[8] S. R. Kasiviswanathan and M. V. Gandhi, "A class of exact solutions for the magnetohydrodynamic flow of a micropolar fluid", Int. J. Eng. Sci. 30 (1992) 409-417.

[9] G. Lukaszewicz, Micropolar fluids—theory and applications (Birkhäuser, Boston, 1999).

[10] M. A. Seddeek, "Effects of Hall and ion-slip currents on magneto-micropolar fluid and heat transfer over a nonisothermal stretching sheet with suction and blowing", Proc. R. Soc. Lond. A 457 (2001) 3039-3050.

[11] V. M. Soundalgekar, N. V. Vighnesam and H. S. Takhar, "Hall and ion-slip effects in MHD Couette flow with heat transfer", IEEE Trans. Plasma Sci. 7 (1979) 178-182.

[12] I. Tani, "Steady flow of conducting fluids in channels under transverse magnetic fields, with consideration of Hall effects", J. Aerospace Sci. 29 (1962) 297-305. 